\title{
Polysèmes
}

Revue d'études intertextuelles et intermédiales

\section{"Such a character would be the regeneration of the world » : variations sur le «type " grec dans l'œuvre de Walter Pater}

\section{Anne-Florence Gillard-Estrada}

\section{OpenEdition}

\section{Journals}

\section{Édition électronique}

URL : http://journals.openedition.org/polysemes/666

DOI : $10.4000 /$ polysemes.666

ISSN : 2496-4212

Éditeur

SAIT

\section{Édition imprimée}

Date de publication : 1 janvier 2012

Pagination : 25-49

ISSN : 0999-4203

\section{Référence électronique}

Anne-Florence Gillard-Estrada, « «Such a character would be the regeneration of the world » variations sur le « type » grec dans l'œuvre de Walter Pater », Polysèmes [En ligne], 12 | 2012, mis en ligne le 01 mars 2015, consulté le 01 mai 2019. URL : http://journals.openedition.org/polysemes/666 ; DOI : 10.4000/polysemes.666

Ce document a été généré automatiquement le 1 mai 2019.

Polysèmes 


\title{
"Such a character would be the regeneration of the world": variations sur le "type " grec dans l'œuvre de Walter Pater
}

\author{
Anne-Florence Gillard-Estrada
}

En 1864, peu après avoir été nommé fellow à Brasenose College, Walter Pater composa un essai intitulé « Diaphaneitè » qu'il lut aux membres de la Old Mortality Society, société d'étudiants de l'Université d'Oxford à laquelle il appartenait. Dans ce texte, Pater vise à définir un «type », personnage diaphane incarnant le modèle d'une culture individuelle idéale. L'essai est à situer dans le contexte de discussions, au sein de la Société, suscitées par la lecture de l'ouvrage de Carlyle On Heroes, Hero-Worship and Heroism in History (1841). La question posée ici par Pater est de savoir quelle serait la meilleure incarnation du " héros ", au sens de Carlyle, dans le monde moderne. Pater propose ainsi à son tour sa propre version de la figure révolutionnaire susceptible d'être le véritable agent du renouveau : "A majority of such would be the regeneration of the world $»^{1}$, mais il oppose son «type » aux « héros » évoqués par Carlyle. Or cette figure grecque, rémanente dans l'ensemble de son œuvre, se caractérise par sa dualité profonde : incarnation d'un élan vital et promesse d'une renaissance que Pater ne cesse d'appeler de ses vœux, elle est aussi, et indissociablement, associée à des images de plus en plus mortifères dans sa production. C'est cette double polarité que l'on voudrait ici mettre en lumière.

2 Le personnage évoqué dans «Diaphaneitè » est ainsi doté d'une force intellectuelle présente en lui comme une réminiscence d'une culture oubliée: "A magnificent intellectual force is latent within it. It is like the reminiscence of a forgotten culture that once adorned the mind; as if the mind of one $\varphi \imath \lambda \circ \sigma \circ \varphi \eta \sigma \alpha \varsigma \pi \circ \tau \varepsilon \mu \varepsilon \tau \varepsilon \rho \circ \tau \circ \varsigma$, fallen into a new cycle, were beginning its spiritual progress over again » (D 254-55). La citation en grec, inventée par Pater, n'est pas traduite, car elle participe d'une véritable stratégie: elle signifie «ayant jadis philosophé avec amour» et s'inspire d'un passage du Phèdre de 
Platon, où le philosophe est celui qui «a aimé les jeunes gens d'un amour philosophique " ${ }^{2}$. L'âme d'un philosophe - qui pourrait être Platon - se serait réincarnée dans ce personnage diaphane. Ainsi, alors que Pater se donne pour tâche, à l'instar de Carlyle et de Matthew Arnold, de redéfinir la notion de "culture », il inscrit son personnage dans une culture qui donne une place centrale au beau corps masculin et à l'eros socratique, et qui privilégie la relation amoureuse entre maître et disciple, tout en mettant lui-même en pratique cette culture " grecque ». À cet égard, il importe de noter que cet essai fut tout d'abord lu à haute voix devant un cercle. Les nombreux déictiques semblent indiquer la présence concrète de cet être diaphane dans une assemblée à laquelle Pater paraît faire constamment référence par son utilisation des mots « here » et « us ». Pater semble ainsi s'adresser à un interlocuteur bien réel, un membre de son auditoire qui l'aurait inspiré. De fait, le destinataire bien réel était sans doute Charles Lancelot Shadwell, son élève, puis son ami intime et son éditeur posthume (Studies in the History of the Renaissance lui fut dédicacé). Pater fait ensuite allusion à la relation, chère à Platon, d'engendrement spirituel entre l'enseignant et son élève : « that receptivity, which professors often strive in vain to form, is engendered here less by wisdom than by innocence » (D 255). Pater réinterprète lui aussi la relation pédagogico-amoureuse entre le maître et son disciple ${ }^{3}$.

3 Ce «type » a des traits grecs : " It has something of the clear ring of the antique. Perhaps it is nearly always found with a corresponding outward semblance » (D 255-56). La beauté de cet être a pour modèle celle des statues antiques. Pater évoque la passivité du personnage, trait qui le distingue du héros carlylien caractérisé par son énergie, établissant notamment une analogie avec le caractère asexué des sculptures grecques: "The beauty of the Greek statues was a sexless beauty: the statues of the gods had the least traces of sex. Here there is a moral sexlessness, a kind of impotence, an ineffectual wholeness of nature, yet with a divine beauty and significance of its own » (D 257). L'indifférence du personnage est assimilée à une forme d'androgynie autant morale que physique, thème qui devint cher à Pater par la suite. Sa nature "révolutionnaire " consiste en effet en une transparence et une absence d'action. Il est aussi défini par une sorte de calme et de simplicite : « the character we have before us is a kind of prophecy of this repose and simplicity» (D 254). Pater s'inspire ici des qualités que l'historien d'art Winckelmann attribuait à la statuaire grecque. À l'image des statues grecques, cet être est comme un vestige du monde antique : «Such a character is like a relic from the classical age, laid open by accident to our alien modern atmosphere » (D 255-56). Il est associé non seulement au diaphane, mais aussi au cristal et à la pierre.

4 Pater souligne aussi la jeunesse de ce type, dont il faut contempler la beauté avant qu'elle se flétrisse avec l'âge adulte : "Often the presence of this nature is felt like a sweet aroma in early manhood. Afterwards, as the adulterated atmosphere of the world assimilates us to itself, the savour of it faints away " (D 258). Apparaît ici une tension que l'on retrouve chez de nombreux personnages historiques ou fictifs de son œuvre. Une note tragique vient en effet assombrir ses propos sur ce type diaphane apollinien. C'est une figure sacrificielle, un avatar du dieu Dionysos Zagreus mis en pièces: «Poetry and poetical history have dreamed of a crisis, where it must needs be that some human victim be sent down into the grave. These are they whom in its profound emotion humanity might choose to send ( (D 258). Ceci reflète une constante oscillation chez Pater entre, d'une part, la contemplation de la beauté de l'être masculin, objet de désir, sujet d'inspiration et protagoniste d'une relation pédagogique, et d'autre part le sentiment de l'interdit, souvent traduit par une mise à mort sacrificielle et esthétisée du jeune homme. 
5 En janvier 1867, le second essai de Pater, « Winckelmann $»^{4}$, dans lequel il transpose les thématiques de "Diaphaneitè », paraît dans la Westminster Review. Le périodique choisi permettait aux auteurs d'exprimer des idées plus radicales, notamment parce que les essais étaient souvent des comptes-rendus de publications plus ou moins récentes; en l'occurrence il s'agit du compte-rendu de l'ouvrage Geschichte der Kunst des Altertums de Winckelmann traduit en anglais en 1850 par G.H. Lodge. Ces essais étant publiés sous l'anonymat, Pater pouvait sans crainte transmettre son esthétique centrée sur le corps masculin, par exemple en reprenant à son compte des citations en allemand ou en grec, parfois non traduites, ou des extraits de textes d'un autre auteur. Le portrait historique était un moyen pour Pater de cacher sa voix auctoriale et de légitimer sa propre sensibilité.

6 Son propos est ainsi de montrer que Winckelmann a inauguré une nouvelle approche de la critique d'art, qu'il qualifie de nouveau « sens ", et ce, dans des « conditions » qu'il faut à ses yeux "reproduire». Winckelmann aurait transmis une tradition culturelle et critique grâce à son "tempérament », et Pater s'attache à montrer le rôle central qu'il a joué dans la culture occidentale en évoquant sa vie et en étudiant sa critique d'art, principalement sa conception des sculptures grecques. Pater reprend alors le paradigme socratique évoqué dans "Diaphaneitè » dont il réécrit presque mot pour mot certains passages. Mais ici, c'est en Winckelmann que se serait réincarnée l'âme du philosophe, habité non plus par une "culture ", mais par un "savoir» oublié : "[Winckelmann] seems to realise that fancy of the reminiscence of a forgotten knowledge hidden for a time in the mind itself : as if the mind of one, lover and philosopher at once in some

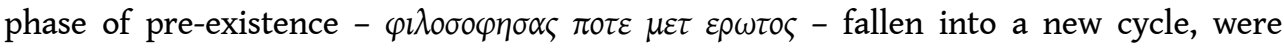
beginning its intellectual career over again » (W 155). Et Winckelmann aurait découvert le véritable «esprit grec» dans des «conditions " particulières qu'il définit comme une affinité avec ce qu'il appelle le «monde du Lysis» de Platon. Il évoque ainsi l'attrait de Winckelmann pour le contexte platonicien de la palestre. Selon lui, la contemplation de la beauté des jeunes gymnastes, chez Platon, est indissociable de la quête philosophique, et comme il l'explique à plusieurs reprises dans son essai, l'esthétique et la critique d'art de Winckelmann s'inspiraient de ce «monde» puisque celui-ci mettait au premier plan la beauté plastique masculine dans ses ouvrages. Or Pater donne aussi une clé de son esthétique personnelle : la recherche de la beauté s'effectue tout autant dans l'art que dans la vie elle-même, principe qu'il place par la suite au cœur de sa Préface et de sa célèbre Conclusion du volume The Renaissance. Car, toujours selon Pater, l'enthousiasme de Winckelmann devant la beauté de la forme masculine le conduit à s'extasier tout autant devant ses jeunes amis que devant les sculptures antiques. Ses "amitiés» passionnées font de lui un "païen " - le terme "pagan» revient souvent - tourné vers une autre époque. Citant des lettres « enfiévrées » que Winckelmann avait envoyées à de jeunes amis, Pater précise que celui- ci considère ce type d'amitié comme plus noble et plus spirituel que l'amour pour une femme. Et ce sont ces amitiés qui lui auraient permis de discerner le véritable esprit de la sculpture grecque :

This key to the understanding of the Greek spirit Winckelmann possessed in his own nature, itself like a relic of classical antiquity, laid open by accident to our alien, modern atmosphere. To the criticism of that consummate Greek modelling he brought not only his culture but his temperament. [...] This temperament he nurtured and invigorated by friendships which kept him always in direct contact with the spirit of youth. (W 175-76) 
7 Le Beau idéal est alors incarné par des représentations qui sont toutes caractérisées par leurs qualités apolliniennes. La statue d'un athlète, beau jeune homme qui rappelle l'être diaphane, est le parfait exemple de ces êtres sculptés qui ont tant ému Winckelmann et Pater après lui : «[T]he adorante of the museum of Berlin, a youth who has gained the wrestler's prize, with hands lifted and open, in praise for the victory. Fresh, unperplexed, it is the image of man as he springs first from the sleep of nature, his white light taking no colour from any one-sided experience » (W 174-75). Ce tout jeune homme est décrit comme blanc et serein. Et le traitement est le même pour les jeunes cavaliers qui composent la frise du Parthénon: "This colourless, unclassified purity of life, with its blending and interpenetration of intellectual, spiritual, and physical elements » (W 174). Puis il fait allusion à l'importance de la figure de l'hermaphrodite pour Winckelmann : le Beau idéal est marqué par la tendance à mêler les traits de la beauté masculine et de la beauté féminine afin de mieux représenter la jeunesse. Par le truchement de la critique d'art de Winckelmann, Pater affirmait sa propre prédilection pour la figure de l'androgyne :

In dealing with youth, Greek art betrays a tendency even to merge distinctions of sex. The Hermaphrodite was a favourite subject from early times. It was wrought out over and over again, with passionate care, from the mystic terminal Hermaphrodite of the British Museum, to the perfect blending of male and female beauty of the Louvre. ${ }^{5}$

8 Pater s'inspirait des discussions de Hegel et de Winckelmann sur la sculpture grecque, qui représente l'homme de façon idéalisée, loin de tout particularisme et de toute anecdote. Il met ainsi en avant les notions de noble simplicité (edle Einfahlt) et de calme grandeur ( stille Grösse) invoquées par Winckelmann pour qualifier les statues grecques ${ }^{6}$. En outre, il fait siennes les théories winckelmanniennes sur la jeunesse: "Now, as the spirit of culture is much more ardent in youth than in manhood, the instinct [for beauty in art] must be exercised and directed to what is beautiful, before that age is reached, at which one would be afraid to confess that one had no taste for it» (W 153). Cependant, on trouve là encore un paradoxe. La promesse d'une renaissance de l'esprit grec s'incarne en effet dans ces statues qui furent exhumées à la Renaissance. Mais Pater associe également à ce motif des « relics ", vestiges du monde antique perdu, l'image du corps mort dans la tombe :

Winckelmann [...] divines the temperament of the antique world, and that in which it had delight. It has passed away with that distant age, and we may venture to dwell upon it. [...] A perfect world, if the gods could have seemed for ever only fleet and fair, white and red! Let us not regret that this unperplexed youth of humanity, satisfied with the vision of itself, passed, at the due moment, into a mournful maturity: for already the deep joy was in store for the spirit, of finding the ideal of that youth still red with life in the grave. (W 166-67)

Dans ce monde antique presque édénique et lointain que l'historien d'art allemand désirait retrouver, la beauté - toujours jeune et masculine - telle que la conçoit Pater ici est celle d'un corps décrit comme rouge et blanc. La promesse de la renaissance prend en outre la forme d'un cadavre enfoui et bientôt exhumé. Le motif du corps dans la tombe fait ainsi écho à celui des statues découvertes, ces « relics » qui permettent à l'idéal grec perdu de renaître et à Winckelmann de le retrouver : « Suddenly he is in contact with that life, still fervent in the relics of plastic art» (W 146). Le corps blanc, lumineux et poli auquel il est fait ici allusion est celui des figures de marbre qui reflètent l'élan apollinien. Mais c'est aussi un corps rouge, un cadavre encore plein de vie, qui évoque le 
dionysiaque. Dans le même temps, Pater effectue une analogie entre l'expérience de Winckelmann et la régénération que connut la Renaissance. La redécouverte, à cette période, des fragments de l'Antiquité est équivalente à des retrouvailles avec le monde des sens qui sont décrites comme une fièvre ou un mal: «When the actual relics of the antique were restored to the world, in the view of the Christian ascetic it was as if an ancient plague-pit had been opened. All the world took the contagion of the senses » (W 180). Ainsi, lorsqu'il découvre ces objets, Winckelmann s'embrase d'un feu libérateur :

[W] can hardly imagine how deeply the human mind was moved, when, at the Renaissance, in the midst of a frozen world, the buried fire of ancient art rose up from under the soil. Winckelmann here reproduces for us the earlier sentiment of the Renaissance. On a sudden the imagination feels itself free. (W 146)

Et une véritable mania divine, inspiration ou possession, s'empare alors de lui. Il est dépeint comme un homme en proie au trouble, une fièvre de type dionysiaque s'emparant de lui lorsqu'il contemple la sérénité apollinienne des statues lisses des jeunes athlètes idéalisés. Pater semble même faire de la mort de Winckelmann une conséquence de ce transport, mort qu'il décrit comme presque désirée par lui et par les dieux. Décidément, les figures du changement semblent pour Pater promises à une mort quasisacrificielle.

11 Le propos de Pater variait souvent selon le lectorat et la politique des différentes revues ou maisons d'édition dans lesquelles il publiait. À partir de 1869, Pater publie d'autres essais, signés cette fois, dans la Fortnightly Review. Un tournant s'opère ici : il peut choisir le sujet de ses essais, notamment la critique d'art et les arts visuels, et il s'intéresse tout particulièrement à certains artistes et écrivains de la Renaissance. Dès lors, il peut exprimer ses idées personnelles sans passer par le prétexte du compte-rendu et sans taire son nom. Portrait historique et portrait imaginaire fusionnent dans ce mode d'écriture qu'il avait adopté dès son essai « Diaphaneitè ». Et les portraits biographiques qu'il brosse fonctionnent comme des sujets qui lui permettent de traiter de thèmes scandaleux. Car en prenant le masque de ces personnages historiques dont il adopte les idées et auxquels il s'identifie tout en s'affirmant comme auteur, Pater abordait certaines thématiques alors sujettes à controverses, comme la passion de Michel-Ange pour Tommaso Cavalieri.

Dans « Leonardo da Vinci » (1869), Pater, s'inspirant de Matthew Arnold, entreprend de définir le rôle et la fonction du critique d'art et s'affirme en tant que critique en se définissant au passage comme "a lover of strange souls $»^{7}$. Il se donne pour tâche de définir ce qui fait le "génie » de cet artiste, «the possessor of some unsanctified and secret wisdom» («Leonardo » 78). Il suggère que le peintre aurait été inspiré pour sa création artistique par son élève Giacomo Salai, qui donna ses traits androgynes au type de beauté du Vinci, qualifiée à plusieurs reprises de " exotic ", " curious » ou "strange ». Pour Pater, ce type réapparaît dans ses dessins et ses tableaux et en retour, ses élèves se seraient inspirés de leur maître et auraient perpétué une tradition esthétique qui mêle les thèmes chrétiens et grecs. Or les descriptions par Pater des tableaux de Vinci reflètent une esthétique très " fin de siècle »: les figures androgynes du Bacchus-Saint Jean ou de la Joconde sont ainsi associées à une beauté mauvaise et inquiétante. Surtout, le Vinci a selon lui transmis un type de beauté androgyne; et il évolue dans une confrérie masculine où le désir entre hommes est le moteur de la création artistique et est à l'origine d'une sensibilité esthétique que Pater souhaite à son tour valoriser.

Dans son essai de 1871, «Pico della Mirandola », Pater explique à nouveau sa conception de la Renaissance, considérée comme une esthétique de l'hybride, un mélange entre 
l'héritage païen et la tradition chrétienne. Pour lui, ce qui faisait la valeur des Néoplatoniciens de Florence était leur tentative de réconcilier ces deux traditions. Il a alors recours à une image curieuse pour symboliser la nouvelle esthétique qui surgit alors, « $[T]$ he anemone with its concentric rings of strangely blended colour [...]. Just such a strange flower was that mythology of the Renaissance, which grew up from the mixture of two traditions, two sentiments, the sacred and the profane » (« Pico» 37). Le choix de cette fleur est étrange à plus d'un titre. Car dans la mythologie grecque, elle surgit du sang qui s'écoule du corps d'Adonis. L'image, même fugitive, du cadavre du beau jeune homme ne peut manquer de surgir à l'esprit du lecteur.

Aux yeux de Pater, Pic de la Mirandole incarne véritablement ce travail de réconciliation emblématique qui s'opère à la Renaissance. Pater choisit en effet de s'attarder sur sa vie et son œuvre car, comme il l'explique, « his own story is a sort of analogue or visible equivalent to the expression of this purpose in his writings » (« Pico » 27). Il reprend alors le thème de l'amitié entre hommes, décrivant par exemple la rencontre à Florence entre Marsile Ficin et Pic de la Mirandole en des termes qui évoquent un échange spirituel, une initiation ésotérique, mais aussi une fascination quasi érotique. Le jeune Pic surgit ainsi de manière providentielle alors que Marsile Ficin met un point final à sa traduction de Platon, censée faire revivre la pensée du philosophe grec - « to resuscitate the knowledge of Plato among his fellow-citizens » («Pico » 27) - et la scène se déroule d'ailleurs devant le buste de celui-ci. Le spectacle esthétique qu'offre Pic est pour Ficin comme un réveil à la fois mystique et intellectuel puisque, selon Pater, ses yeux jusque-là mi-clos s'ouvrent alors sur la beauté physique. Pater évoque d'ailleurs plus loin le discours de Pic de la Mirandole sur l'ascension de l'âme depuis la beauté visible vers la beauté invisible, discours qui permettait en fait à Pater de valoriser une fois encore la beauté masculine. Pic est ainsi décrit comme un beau jeune homme, hybride de dieu antique et d'ange chrétien : «a young man, not unlike the archangel Raphael, [...] or Mercury " (« Pico » 29). À nouveau, il met en avant le thème de la relation à la fois éducative et amoureuse entre le maître et l'élève. Et il retient tout particulièrement du texte de Thomas More dont il s'inspire l'exceptionnelle impression de beauté qui émane de ce beau jeune homme presque androgyne, dont certaines parties du corps sont décrites avec une attention très sensuelle :

And now the work was completed, the door of the mystical temple lay open to all who could construe Latin, and the scholar rested from his labour: when there was introduced into his study, where a lamp burned continually before the bust of Plato, as other men burned lamps before their favourite saints, a young man fresh from a journey, 'of feature goodly and shape seemly and beauteous, of stature goodly and high, of flesh tender and soft, his visage lovely and fair, his colour white intermingled with comely reds, his eyes grey, and quick of look, his teeth white and even, his hair yellow and abundant,' and trimmed with more than the usual artifice of the time. (" Pico» 28)

Puis, après voir évoqué la vie et l'œuvre de Pic, Pater s'attarde sur la description de sa dépouille, reprenant une nouvelle fois l'image du corps rouge du jeune homme dans la tombe: "And so, while his actual work has passed away, yet his own qualities are still active, and himself remains, as one alive in the grave [...] and with that sanguine, clear skin, decenti rubore interspersa » ( Pico » 28), c'est-à-dire, selon le texte de More cité par Pater, «intermingled with comely reds». Certes, Pic apporte cette promesse d'une transmission de la culture néoplatonicienne. Cependant, Pater évoquait au début de l'essai une réécriture du mythe d'Apollon par Heinrich Heine, premier avatar des transpositions mythologiques tardives de Pater qui suggèrent invariablement la 
réconciliation impossible entre les deux religions. Ici, le dieu grec aux traits androgynes et à la beauté ensorceleuse réapparait à l'époque médiévale mais est soumis à une mort atroce, puis est enterré. Toutefois, lorsque les hommes veulent déterrer son cadavre, la tombe est vide, ce qui suppose l'impossibilité d'un quelconque renouveau. À cause de cette digression initiale, la thématique de la renaissance, symbolisée par le cadavre encore jeune et rouge de vie, commence dès cet essai à s'assombrir : une hantise de plus en plus vive de la mort, doublée de la certitude qu'aucune renaissance n'est possible, s'attache à cette figure du jeune homme et surtout au topos du dieu grec sacrifié. La thématique de la mort violente de tels agents du changement, présente dès " Diaphaneitè » et "Winckelmann ", réapparaît donc ici de manière plus radicale et plus tragique. Certes, l'essai évoque ensuite le rôle joué par l'homme Pic de la Mirandole et par son œuvre dans la diffusion d'une culture socratique et néoplatonicienne que Pater voulait légitimer mais qui s'avérait de plus en plus incompatible avec les valeurs de son époque, comme il commençait à en faire l'amère expérience. Peut-être Pater laissait-il déjà transparaitre, à travers cette mort qui ne contient aucune promesse de renaissance, son pessimisme quant à la possibilité de raviver cette culture philosophique et esthétique.

En 1873, Pater adopte un nouveau format de publication pour exprimer ses idées. Pour la première édition du volume Studies in the History of the Renaissance publié chez Macmillan, il rassemble certains des essais qu'il avait publiés, auxquels il ajoute de nouveaux textes. Dans ce recueil, son projet est de proposer une vision nouvelle de la Renaissance et de la Grèce. Il bat en brèche les conceptions de deux penseurs contemporains de poids, mais sans jamais les nommer : celles de Ruskin, chantre de l'art primitif chrétien, qui vitupéra dans certains de ses essais l'art de la Renaissance, considérée comme une période de déclin moral et culturel ; et celles de Matthew Arnold, dont il subvertit les propos sur la et le critique d'art lorsqu'il définit sa propre notion de "aesthetic criticism» et qu'il développe sa théorie du beau et des impressions. Il approfondit en outre sa thématique de la Renaissance et de la Grèce comme espaces de conflit pour l'apollinien et le dionysiaque ou pour le classicisme et le romanticisme tels qu'il les conçoit. En effet, selon Pater, la Renaissance consistait en une fusion entre la sérénité des Grecs et la dimension plus tourmentée et plus enfiévrée propre à « l'esprit romantique ».

Dans la Préface qu'il rédige pour le volume, il justifie le choix de ce thème de la Renaissance en expliquant qu'elle fut l'époque où survint un renouveau de l'« esprit grec ", qu'il décrit comme une sorte de débordement de l'être et une libération de l'esprit humain. Pater estime par ailleurs que le « critique esthétique » doit considérer l'art selon son "tempérament ", mot qui, dans l'essai sur «Winckelmann » inséré à la fin de son recueil, est à resituer dans ce contexte homoérotique inspiré d'une lecture platonicienne très personnelle évoqué plus haut. Il reprend en outre dans la Préface l'idée selon laquelle le critique doit s'intéresser à la beauté sous toutes ses formes : beauté présente dans l'art ou dans la nature, mais aussi beauté de la forme humaine. Et dans la Conclusion qu'il apporte à son volume, il inscrit un passage de son essai de 1868, «Poems by William Morris ", dans lequel il énonce son célèbre credo esthétique : parce qu'il est confronté au flux implacable, l'homme doit rechercher chaque instant de beauté, aussi bien dans l'art que dans la vie. Or la première publication de Studies in the History of the Renaissance de 1873 suscita de violentes attaques contre le propos de l'ouvrage. Et à la même époque, des scandales concernant les relations de Pater avec certains de ses étudiants mirent un frein à sa carrière universitaire. C'est pourquoi Pater jugea préférable de supprimer la Conclusion pour la seconde édition de 1877 intitulée The Renaissance: Studies in Art and 
Poetry, tant le credo esthétique de celle-ci apparaissait comme hédoniste aux yeux de certains de ses contemporains. Or Pater se décida à rétablir celle-ci pour la troisième édition de 1888, expliquant dans une note qu'il l'avait ôtée afin de ne pas égarer un lectorat qu'il prend soin de définir comme jeune et masculin: "I conceived it might possibly mislead some of those young men into whose hands it might fall $»^{8}$. Il n'avait en fait jamais abandonné son projet de valoriser une esthétique centrée sur le corps masculin, adoptant au contraire durant toute sa carrière des stratégies de réécriture visant à réaffirmer les thématiques des débuts mais tout en intériorisant de plus en plus l'opprobre qui entourait son idéal grec.

18 À partir de 1876, Pater rédige des essais très documentés sur la religion et les mythes grecs, qui s'appuient notamment sur les avancées philologiques, anthropologiques et archéologiques de l'époque et dans lesquels il propose une approche assez novatrice de ceux-ci. Il établit par exemple un lien entre Dionysos et le Christ et s'intéresse à certains aspects du culte dionysiaque jusque-là souvent ignorés: la souffrance, le sacrifice humain, l'androgynie, la violence rituelle ou encore l'importance du corporel et du sensuel. Ces éléments témoignent de l'intérêt de Pater pour certains caractères sombres, tragiques ou licencieux de la religion grecque, plaçant ainsi ces études aux antipodes de la vision de Matthew Arnold d'une culture grecque lumineuse et édulcorée. Parallèlement, dès 1880 , il se penche à nouveau sur l'étude de la sculpture grecque dans des essais très informés par les découvertes archéologiques récentes. Il publie ces différents textes dans des revues, mais ceux-ci ne parurent qu'en 1895 dans le volume posthume Greek Studies, qui contenait aussi ses essais sur les mythes ${ }^{9}$. En effet, il n'osa pas publier ceux-ci de son vivant, car la Grèce était alors devenue synonyme d'immoralité pour beaucoup. Dans ces essais sur la statuaire, il reprend à son compte le type de critique d'art pratiqué selon lui par Winckelmann : le regard désirant du critique semble ainsi caresser les athlètes grecs et s'attarder sur leur blanche nudité ${ }^{10}$.

Dans ces mêmes années 1890, il prononce une série de conférences à Oxford sur Platon et la philosophie grecque, publiées en 1893 dans le recueil Plato and Platonism. Il y adopte le point de vue académique du professeur de philosophie qu'il était, et s'inscrit là encore dans une relation pédagogique. Il évoque à plusieurs reprises le motif du couple de l' erastes et l'eromenos, l'amant et l'aimé, et s'attarde aussi sur la figure de Socrate, insistant sur le véritable attachement que celui-ci vouait à ses disciples. Il semble même s'identifier à lui en le présentant comme une victime accusée de corrompre les jeunes gens alors que lui aussi, l'enseignant d'Oxford, fut dénoncé par ses pairs. Dans un passage écrit dans le même style que la Conclusion de The Renaissance, il évoque par exemple un discours de Socrate sur l'immortalité de l'âme, délivré à un jeune auditoire semblable au public auquel lui-même s'adressait :

Those young Athenians whom he was thought to have corrupted of set purpose, he had not only admired but really loved and understood: and as a consequence had longed to do them good, chiefly by giving them that interest which is the first condition of any real power over others. To make Meno, Polus, Charmides, really interested in himself, to help him to the discovery of that wonderful new world here at home-in this effort [...] lay and still lies [...] the central business of education. [...] He had not been able in all cases to expand 'the better self', as people say, in those he influenced. ${ }^{11}$

Ce passage fait penser à la note ajoutée pour justifier le rétablissement de la Conclusion ; et le contenu du savoir que Socrate, selon Pater, désire inculquer à ces jeunes hommes frappe par sa similitude avec l'exhortation de la Conclusion à profiter de chaque 
sensation : " our one chance lies in expanding that interval »" Pater cite ensuite des extraits du Phédon où le vocabulaire employé par Socrate pour garantir l'immortalité de l'âme donne la part belle à la primauté des sensations visuelles et à l'importance des couleurs, passages vraisemblablement choisis pour leur similitude avec le lexique et les images de la Conclusion. Son propos est probablement d'établir un parallèle entre les enseignements que Socrate dispensait à son jeune auditoire et le credo que Pater destinait à son jeune lectorat. Notons qu'une fois encore, les cours de Pater sur les philosophes grecs furent d'abord lus à un public de jeunes étudiants d'Oxford. Malgré les attaques qu'il avait subies dans la presse, en chaire ou à l'Université, Pater reprend donc le projet et les idées exprimées des années auparavant en les transposant à d'autres genres et d'autres modes d'écriture.

21 À partir de 1887, Pater publie de courts textes de fiction dont certains ont pour héros de jeunes hommes. Il en situe le décor dans des pays et des époques différentes, établissant ainsi une distance avec le contexte anglais contemporain. À l'instar des portraits historiques des débuts, ces personnages imaginaires fonctionnent comme de véritables écrans entre Pater et le lecteur ; toutefois, leur caractère autobiographique est manifeste.

En 1892 parut dans la New Review un récit de fiction intitulé « Emerald Uthwart ». Le titre rappelle son premier essai sur l'être diaphane, qu'il comparait à un être de cristal. Pater met en scène un jeune Anglais, Emerald, élève d'une public school du début du XIX siècle, qui est profondément influencé par le paradigme socratique d'éducation et d'amitié : la construction physique et morale du jeune éphèbe anglais s'opère ainsi grâce à la lecture du Phèdre de Platon. L'éveil intellectuel d'Emerald et de l'un de ses camarades est tout d'abord comparé à un éveil amoureux :

In every generation of schoolboys there are a few who find out [...] the beauty and power of literature [...]: and this, in turn, is but the handsel of a beauty and power still active in the actual world, should they have the good fortune, or rather, acquire the skill, to deal with it properly. It has something of the stir and unctionthis intellectual awaking with a leap-of the coming of love. So it was with Uthwart about his seventeenth year. He felt it, felt the intellectual passion, like the pressure outward of wings within him $-\eta \pi \tau \varepsilon \rho o v ~ \delta v v \alpha \mu l \zeta$, says Plato, in the Phaedrus: but again, as some do with everyday love, withheld, restrained himself. ${ }^{13}$

Cet extrait, très proche de certaines phrases de l'essai "Winckelmann ", rappelle la thématique platonicienne: Emerald découvre la passion à la lecture du passage concernant le délire ressenti à la vue de l'aimé. Cependant, Pater suggère assez vite que l'amitié socratique est promise à l'échec. Les deux compagnons partent ainsi à la guerre, mais après un acte d'indiscipline, son ami est exécuté sous ses yeux, Emerald est chassé de l'armée et il meurt peu après d'une ancienne blessure.

Pourtant, cette culture grecque semblait tout d'abord pleine de promesses. Le jeune Emerald est décrit comme un dieu grec réincarné. Il ressemble à l'athlète représenté sur une stèle funéraire qui avait inspiré Pater pour ses études sur la statuaire grecque. Mais c'est surtout lorsqu'il est figé dans la mort qu'il redevient comme une statue antique. Son cadavre est alors contemplé par un médecin, ce qui l'autorise - et par là même Pater, ou encore le lecteur - à s'attarder sur ce corps désirable. Les sculptures grecques avaient constitué un prétexte parfait pour décrire l'émoi ressenti par Winckelmann devant ces corps masculins qui ne le laissaient pas de marbre. Ici, c'est par le biais de la fiction que le désir interdit et la contemplation de la beauté masculine sont exprimés. Cette beauté ne peut être représentée que par l'image obsédante du cadavre du beau jeune homme dans la tombe, nouvelle incarnation tragique de l'idéal grec. Car Emerald évolue dans un 
environnement enrégimenté - notamment cette public school dont l'architecture gothique n'est pas sans évoquer Oxford - où les retrouvailles avec cette culture grecque sont justement impossibles. Le beau corps masculin suscite un trouble esthétique et homoérotique, mais il ne s'agit plus ici que d'une nécrophilie improductive. Car dans ce texte, le lien émotionnel et intellectuel entre deux êtres - un homme plus jeune et un autre plus âgé - est voué au sacrifice et à la mort. Le discours sur l'idéal d'amitié inspiré du modèle grec prend donc des accents pessimistes et ne donne lieu qu'à une fin tragique qui semble refléter le propre état d'esprit de Pater. Plus encore qu'auparavant, la perfection esthétique incarnée par Emerald fait d'emblée de lui une figure sacrificielle.

Dans deux autres Portraits Imaginaires, Pater renoue avec le thème du retour des dieux païens dans un monde médiéval hostile, thème qu'il avait emprunté à Heinrich Heine. Ces réécritures fictionnelles de mythes grecs reprennent les thématiques de prédilection de Pater: le conflit entre paganisme et christianisme, ou, en termes arnoldiens, entre Hébraïsme et Hellénisme ; le paradigme de l'éducation pédagogico-amoureuse ; et le motif $\mathrm{du}$ dieu jeune et beau, figure duelle aux traits androgynes, qui exerce une fascination inexpliquée. "Denys L'Auxerrois» (publié tout d'abord dans le Macmillan Magazine en 1886 puis dans le volume Imaginary Portraits ${ }^{14}$ ) et "Apollo in Picardie » (publié en 1893 dans Harper's New Monthly Magazine puis dans le recueil posthume Miscellaneous Studies ${ }^{15}$ ) semblent tout d'abord annoncer la thématique de la promesse d'une renaissance. En effet, l'arrivée dans la France du Moyen-Âge de Denys et d'Apollyon, avatars de Dionysos et d'Apollon, crée un temps les conditions d'une libération de l'être, d'une renaissance des sens et d'un épanouissement à la fois social, politique et culturel. Sous leur influence, un véritable renouveau artistique s'opère : à rebours de l'évolution de l'histoire de l'art telle que la concevait Ruskin, les édifices médiévaux gothiques redeviennent harmonieux en retrouvant des proportions classiques.

Dans le premier texte, Hermès, un vieux moine, tombe sous le charme de Denys et entreprend d'éduquer à son tour son inspirateur. Mais l'énergie dionysiaque que le passage du jeune homme suscite dans la ville d'Auxerre devient vite incontrôlable. La version plus sanglante du mythe de Dionysos - celle-là même que Pater avait abordée dans ses essais sur la mythologie grecque - est alors transposée dans la fiction. Le jeune homme, confronté à ce monde chrétien qui le rejette, sombre dans la folie et le déchirement. Parallèlement, c'est une véritable atmosphère de violence bachique qui envahit la ville tout entière et Denys, à l'instar de Dionysos Zagreus, est alors perçu comme une victime expiatoire : il est finalement chassé et mis en pièces par les habitants lors d'une procession religieuse. Cependant, le cycle de la souffrance, de la mort, puis de la résurrection qui caractérise le mythe de Zagreus ne fonctionne pas ici : son coeur est enterré au pied d'une croix pour tomber finalement en poussière.

Dans le second portrait, Apollyon est comme une réincarnation du dieu dorien Apollon qui surgit dans une communauté monacale de Picardie. Être beau, mais comportant un versant dionysiaque et destructeur, conformément à son origine mythique ${ }^{16}$, il offre dans un premier temps au Prieur Saint Jean et à Hyacinth, le jeune novice de celui-ci, la possibilité de faire l'expérience d'un éveil intellectuel et d'une plénitude sensuelle retrouvée. Mais là encore la fiction reflète la blessure autobiographique. La suspicion ressentie par les moines envers cette résurgence du monde païen interdit l'appropriation de l'idéal classique généreux qu'il incarne, et ce d'autant plus qu'Apollyon est saisi d'une folie meurtrière : il tue Hyacinth accidentellement et est contraint à l'exil. Toutefois, Pater tient à indiquer que le sang du jeune Hyacinth ne donne naissance à aucune fleur, 
contrairement à la légende mythologique. Le Prieur croit apercevoir des jacinthes, mais elles sont décrites comme des illusions, les fleurs du renouveau n'étant que de vagues teintes bleutées.

Les lectures divergent sur l'interprétation de ce dernier portrait. Peut-être Pater exprimait-il ses craintes devant la tournure païenne et parfois scandaleuse qu'auraient prise chez certains de ses contemporains ou de ses disciples son hellénisme et son culte des impressions ${ }^{17}$. Quoi qu'il en soit, Pater avait voulu montrer dans ses études de mythographie que les mythes grecs étaient en devenir permanent : ils donnaient lieu à une multiplicité de formes et d'interprétations et reflétaient les aspirations profondes de l'homme. Le mythe, à ses yeux, se prête ainsi à de constantes relectures et réécritures, et lui aussi désira s'inscrire dans cette fabrication mythologique. Probablement avait-il voulu exprimer dans ses dernières œuvres de fiction et dans ses propres réécritures mythiques sa conviction de plus en plus profonde que la régénération incarnée par cet être grec qui revient dans un milieu à la fois homosocial et homophobe - l'analogie avec le milieu oxonien de Pater est très nette dans ces trois Portraits - est en fait impossible. Mais c'est son élève et lecteur attentif, Oscar Wilde, qui eut à payer le prix fort pour s'être épris lui aussi de cet idéal grec.

\section{BIBLIOGRAPHIE}

Adams, J.E. Dandies of Desert Saints: Styles of Victorian Masculinity. Ithaca \& London: Cornell UP, 1995.

Brake, Laurel. Walter Pater. Plymouth: Northcote House, 1994.

Dellamora, Richard. Masculine Desire: The Sexual Politics of Victorian Aestheticism. London, Chapel Hill: University of North Carolina, 1990.

D’Hangest, G. Walter Pater : L'homme et l'œuvre. Paris : Didier, 1961.

Dowling, Linda. Hellenism and Homosexuality in Victorian Oxford. Ithaca \& London: Cornell UP, 1994.

Monsman, Gerald. Oxford University's Old Mortality: A Study in Victorian Romanticism. Lewinston, N.Y : The Edwin Mellen Press, 1998.

Pater, Walter. Imaginary Portraits. London: Macmillan, 1887.

Pater, Walter. Plato and Platonism. London: Macmillan, 1893.

Pater, Walter. Miscellaneous Studies. C.L. Shadwell (ed.). London: Macmillan, 1895.

Pater, Walter. Greek Studies. C.L. Shadwell (ed.). London: Macmillan, 1895.

Pater, Walter. Studies in the History of the Renaissance. Donald Hill (ed.). Berkeley: University of California Press, 1980.

Platon. Phèdre (C. Moreschini et P. Vicaire, trads.). Paris : Les Belles Lettres, édition bilingue, 1998. 
Winckelmann, J.J. Gedanken über die Nachahmung der griechischen Werke in der Malerei und Bildhauerkunst; Réflexions sur l'imitation des œuvres grecques en peinture et en sculpture. Paris : Aubier, édition bilingue, 1990.

\section{NOTES}

1. «Diaphaneitè ", Miscellaneous Studies, C.L. Shadwell (ed.), London: Macmillan, 1895, 259. Ciaprès $\mathrm{D}$ dans les références entre parenthèses dans le texte.

2. Platon, Phèdre, trad. C. Moreschini et P. Vicaire, Paris : Les Belles Lettres, édition bilingue, 1998, 64-65.

3. Sur la relecture de Platon par Pater, voir mon article : «Les dialogues de Walter Pater avec Platon : le philosophe et l'amoureux », Cahiers Victoriens et Edouardiens 63 (avril 2006), 387-402.

4. "Winckelmann", Studies in the History of the Renaissance, Donald Hill (ed.), Berkeley: U of California $\mathrm{P}, 1980$. Ci-après $\mathrm{W}$ dans les références entre parenthèses dans le texte.

5. Ce passage fut supprimé dès la seconde édition ; voir The Renaissance, op. cit., 263.

6. J.J. Winckelmann, Gedanken über die Nachahmung der griechischen Werke in der Malerei und Bildhauerkunst; Réflexions sur l'imitation des oeuvres grecques en peinture et en sculpture, Paris : Aubier, éd. bilingue, 1990, 61-62.

7. "Leonardo da Vinci », The Renaissance, op. cit., 78. Comme l'ont démontré Dowling et Dellamora, tous ces adjectifs, ainsi que ceux indiqués plus bas, étaient codés et renvoyaient en fait au thème sulfureux de l'homoérotisme.

8. «Conclusion ", The Renaissance, op. cit., 186.

9. Greek Studies, C.L. Shadwell (ed.), London: Macmillan, 1895.

10. Voir mon article "Walter Pater et le paradoxe de l'idéal grec incarné par la sculpture", Cahiers Victoriens et Edouardiens 59 (avril 2004), 269-281.

11. «Plato and Socrates », Plato and Platonism, London: Macmillan, 1893, 79-80.

12. "Conclusion », The Renaissance, op. cit., 190.

13. « Emerald Uthwart », Miscellaneous Studies, op. cit., 222-23.

14. Imaginary Portraits, London: Macmillan, 1887.

15. Miscellaneous Studies, C.L. Shadwell (ed.), London: Macmillan, 1895.

16. La racine de son nom est celle de mots traduits par « détruire » ou «perte ». Apollyon est par ailleurs une figure démoniaque dans la Bible.

17. C'est la thèse de G. D'Hangest. Voir son ouvrage Walter Pater : L'homme et l'œuvre, Paris : Didier, 1961, 79-82.

\section{INDEX}

oeuvrecitee Denys l'Auxerrois, Diaphaneitè, Emerald Uthwart, Greek Studies, Imaginary Portraits, Plato and Platonism, Studies in the History of the Renaissance, Winckelmann 